\title{
ON THE CONJECTURE OF GACKSTATTER AND LAINE CONCERNING THE DIFFERENTIAL EQUATION
}

$$
\left(w^{\prime}\right)^{n}=\sum_{j=0}^{m} a_{j}(z) w^{j}
$$

Dedicated to Professor M. Ozawa on his sixtieth birthday

By Nobushige Toda

\section{Introduction}

In this paper, we consider the differential equation

$$
\left(w^{\prime}\right)^{n}=\sum_{j=0}^{m} a_{\jmath}(z) w^{\jmath} \quad(0 \leqq m \leqq 2 n)
$$

in the complex plane, where the $a_{j}(z)(\jmath=0,1, \cdots, m)$ are meromorphic in $|z|<\infty$ and $a_{m}(z) \not \equiv 0$. It is said ([3]) that any solution $w(z)$ of (1) is admissible if it is meromorphic in $|z|<\infty$ and satisfies the condition

$$
T\left(r, a_{j}\right)=o(T(r, w)) \quad(j=0, \cdots, m)
$$

for $r \rightarrow \infty$ possibly outside a set $E$ of $r$ of finite linear measure. (From now on, we denote by $E$ any set of $r$ of finite linear measure.) In the sequel, when the condition (2) is satisfied, we simply denote by

as usual (see [5], p. 55).

$$
T\left(r, a_{j}\right)=S(r, w)
$$

Recently, Gackstatter and Laine $([3], \S 3)$ have investigated the differential equation (1) in many cases and conjectured that it does not possess any admissible solution when $1 \leqq m \leqq n-1$. With respect to this conjecture, Ozawa ([9]) proved the following

Theorem A. When $m=1,2$ and 3 , the differential equation (1) for $m \leqq n-1$ does not admit any admissible solution except when

with a constant $\alpha$.

$$
\left(w^{\prime}\right)^{n}=a_{m}(w+\alpha)^{m}
$$

Further, he gave the following

THEOREM B. When $m=1,2$ and 3 , let $\rho$, be the order of $a,(j=0, \cdots, m)$ and suppose that they are finte. Then, any meromorphic solution of (1) for $m \leqq n-1$ is of order at most $\rho$, where

Received November 8, 1982 


$$
\rho=\max \left(\rho_{0}, \rho_{1}, \cdots, \rho_{m}\right) .
$$

His proofs contain a lot of very complicated calculations, which would not be applicable to the general case, but on the other hand, they also contain many good ideas which we can use in the general case.

The purpose of this paper is to show that Theorems A and B hold good for any $m$ and $n$ such that $1 \leqq m \leqq n-1$. It is assumed that the reader is familiar with the notation of Nevanlinna theory (see [5], [8]).

\section{Nonexistence of admissible solutions}

To begin with, we shall give some lemmas for later use.

LEMMA 1. Let $g_{0}$ and $g_{1}$ be meromorphic in $|z|<\infty$ and linearly independent over $C$, and put

(3)

Then, we have

$$
g_{0}+g_{1}=\psi \text {. }
$$

$$
\begin{aligned}
T\left(r, g_{0}\right) \leqq & m(r, \phi)+N\left(r, g_{0}\right)+\bar{N}\left(r, 0, g_{0}\right)+\bar{N}\left(r, 0, g_{1}\right) \\
& +\bar{N}\left(r, g_{0}\right)+\bar{N}\left(r, g_{1}\right)+S(r),
\end{aligned}
$$

where

(4) $S(r)=\left\{\begin{array}{l}O(1) \text {, when } g_{0} \text { and } g_{1} \text { are rational; } \\ O(\log r) \text {, when } g_{0} \text { and } g_{1} \text { are of finite order; } \\ O\left(\log T\left(r, g_{0}\right)+\log T\left(r, g_{1}\right)\right)+O(\log r)(r \rightarrow \infty, r \in E) \text {, the other cases. }\end{array}\right.$

Proof. Differentiating both sides of (3), we have

from which we obtain

$$
g_{0}^{\prime}+g_{1}^{\prime}=\psi^{\prime}
$$

$$
\frac{g_{0}^{\prime}}{g_{0}} g_{0}+\frac{g_{1}^{\prime}}{g_{1}} g_{1}=\phi^{\prime} \text {. }
$$

From (3) and (5), we get

Then,

$$
g_{0}=\left|\begin{array}{ll}
\psi & 1 \\
\psi^{\prime} & g_{1}^{\prime} / g_{1}
\end{array}\right| /\left|\begin{array}{ll}
1 & 1 \\
g_{0}^{\prime} / g_{0} & g_{1}^{\prime} / g_{1}
\end{array}\right| .
$$

using

$$
\begin{aligned}
m\left(r, g_{0}\right) \leqq & m(r, \phi)+m\left(r, g_{1}^{\prime} / g_{1}-\psi^{\prime} / \psi\right)+m\left(r,\left(g_{1}^{\prime} / g_{1}-g_{0}^{\prime} / g_{0}\right)^{-1}\right)+O(1) \\
\leqq & m(r, \phi)+m\left(r, g_{1}^{\prime} / g_{1}\right)+m\left(r, \psi^{\prime} / \psi\right)+m\left(r, g_{1}^{\prime} / g_{1}-g_{0}^{\prime} / g_{0}\right) \\
& +N\left(r, g_{1}^{\prime} / g_{1}-g_{0}^{\prime} / g_{0}\right)+O(1),
\end{aligned}
$$

$$
\begin{aligned}
T(r, \phi) \leqq & T\left(r, g_{0}\right)+T\left(r, g_{1}\right)+O(1), \\
& \leqq m(r, \psi)+\bar{N}\left(r, 0, g_{0}\right)+\bar{N}\left(r, g_{0}\right)+\bar{N}\left(r, 0, g_{1}\right)+\bar{N}\left(r, g_{1}\right)+S(r),
\end{aligned}
$$

where $S(r)$ is defined by (4), which we can obtain from Lemma 2([8], pp. 6263). Adding $N\left(r, g_{0}\right)$ to both sides, we obtain the result. 
LEMMA 2. Let $f, b_{0}, \cdots, b_{k}$ be meromorphic in $|z|<\infty$ such that $b_{k} \neq \equiv$. Then, we have the following inequalities:

(i) $\quad m\left(r, \sum_{\jmath=0}^{k} b_{\jmath} f^{\jmath}\right) \leqq k m(r, f)+\sum_{\jmath=0}^{k} m\left(r, b_{\jmath}\right)+O(1)$;

(ii) $\quad T\left(r, \sum_{j=0}^{k} b_{\jmath} f^{\jmath}\right) \leqq k T(r, f)+\sum_{j=0}^{k} T\left(r, b_{j}\right)+O(1)$;

(iii) $\left|T\left(r, b_{k}\left(f+b_{0}\right)^{k}\right)-k T(r, f)\right| \leqq T\left(r, b_{k}\right)+k T\left(r, b_{0}\right)+O(1)$.

(see [4], p. 46).

We can easily prove (i) and (ii) by the mathematical induction and (iii) from the fundamental properties of the characteristic function of meromorphic functions. More precise relations are known (see ([7]), but this lemma is good enough to prove our theorems.

LeMma 3. Let $f(z)$ be transcendental meromorphic in $|z|<\infty, P(z)$ and $Q(z)$ differential polynomials in $f(z)$ such that

$$
(f(z))^{n} P(z)=Q(z)
$$

in $|z|<\infty$. If the degree of $Q(z)$ is at most $n$, then

$$
m(r, P)=S(r, f)
$$

([2], Lemma 2, see also [5], Lemma 3.3).

LEMMA 4. Suppose that the differential equation (1) possesses an admissible solution $w(z)$ for $1 \leqq m \leqq n-1$. Then,

$$
\begin{gathered}
N(r, w)=S(r, w), \quad N\left(r, w^{\prime}\right)=S(r, w) ; \\
n T\left(r, w^{\prime}\right) \leqq m T(r, w)+S(r, w) ;
\end{gathered}
$$

(8) for a meromorphic function $a(z)$ in $|z|<\infty$, if

then

$$
T(r, a)=S\left(r, w^{\prime}\right),
$$

$$
T(r, a)=S(r, w),
$$

In fact, (6) is given in [3], p. 266. Applying Lemma 2 to (1), we obtain (7). (8) is trivial from (7).

THEOREM 1. The differential equation (1) does not possess any admissible solutions for $1 \leqq m \leqq n-1$ except when $n-m$ is a divisor of $n$ and (1) has the following form:

$$
\left(w^{\prime}\right)^{n}=a_{m}(w+\alpha)^{m} \quad(\alpha: \text { constant })
$$


Proof. We shall prove this theorem dividing into three cases.

I. For $2 \leqq m \leqq n-1$, the differential equation (1) does not possess any admissible solutions except when it has the form

$$
\left(w^{\prime}\right)^{n}=a_{m}(w+b)^{m},
$$

where $b$ is meromorphic in $|z|<\infty$.

In fact, suppose that (1) does not have the form (10) and admits an admissible solution $w=w(z)$ when $2 \leqq m \leqq n-1$. We rewrite the righthand side of (1) as follows :

$$
\sum_{j=0}^{m} a_{j} w^{\jmath}=a_{m}(w+b)^{m}+\sum_{j=0}^{\mu} b_{\jmath} w^{\jmath} \quad\left(0 \leqq \mu \leqq m-2, b_{\mu} \not \equiv 0\right)
$$

where

$$
b=a_{m-1} / m a_{m} \text { and } b_{\jmath} \text { is a rational function of } a_{\jmath}, a_{m-1} \text { and } a_{m} .
$$

By the choice of $b, \mu$ is not greater than $m-2$. We note that

$$
T(r, b)=S(r, w) \quad \text { and } T\left(r, b_{j}\right)=S(r, w) .
$$

Here, we apply Lemma 1 to

$$
g_{0}=-a_{m}(w(z)+b)^{m}, \quad g_{1}=\left(w^{\prime}(z)\right)^{n} \quad \text { and } \quad \phi=\sum_{j=0}^{\mu} b_{j}(w(z))^{\jmath} .
$$

Then,

$$
g_{0}+g_{1}=\psi
$$

We note that (i) $\phi \not \equiv$ and (ii) $g_{0}$ and $g_{1}$ are linearly independent over C. First, we prove (i). If $\mu=0, \phi=b \neq 0$ by the assumption. When $\mu \geqq 1$, suppose $\phi \equiv 0$, then we have

$$
b_{\mu} w^{\mu}=-\sum_{j=0}^{\mu-1} b_{\jmath} w^{\jmath}
$$

Here, $b_{\mu} \neq 0$ and by Lemma 2(ii).

$$
\mu T(r, w) \leqq(\mu-1) T(r, w)+S(r, w),
$$

which is absurd. That is, $\phi \not \equiv 0$.

Next, we prove (ii). Suppose that $g_{0}$ and $g_{1}$ are linearly dependent over C:

$$
\alpha g_{0}+\beta g_{1} \equiv 0 \quad(|\alpha|+|\beta| \neq 0, \alpha, \beta \in C) .
$$

Then, from (1) and (12), we have

$$
\alpha a_{m} w^{m}+\alpha a_{m-1} w^{m-1}+\cdots+\alpha a_{m} b^{m}=\beta a_{m} w^{m}+\beta a_{m-1} w^{m-1}+\cdots+\beta a_{0} .
$$

As $1, w, \cdots, w^{m}$ are linearly independent over the field of meromorphic functions $a(z)$ satisfying $T(r, a)=S(r, w)$ (this fact can be easily proved as in the case (i)), we obtain $\alpha=\beta$, so that from (11) and (12), $\alpha \phi \equiv 0$. From (i), $\phi \neq 0$ and so 
$\alpha=0$, which is a contradiction. That is, $g_{0}$ and $g_{1}$ must be linearly independent over $C$.

Now, by Lemma 1 ,

$$
\begin{aligned}
T\left(r, g_{0}\right) \leqq & m(r, \phi)+N\left(r, g_{0}\right)+\bar{N}\left(r, 0, g_{0}\right)+\bar{N}\left(r, 0, g_{1}\right) \\
& +\bar{N}\left(r, g_{0}\right)+\bar{N}\left(r, g_{1}\right)+S(r) .
\end{aligned}
$$

Here, we use the following estimates:

$$
\begin{aligned}
& T\left(r, g_{0}\right)=m T(r, w)+S(r, w) \quad \text { (by Lemma 2(iii)); } \\
& T\left(r, g_{1}\right)=n T\left(r, w^{\prime}\right) \leqq m T(r, w)+S(r, w) \quad \text { (by Lemma 4); } \\
& N\left(r, g_{0}\right)=S(r, w), \quad N\left(r, g_{1}\right)=S(r, w) \quad \text { (by Lemma 4); } \\
& \bar{N}\left(r, 0, g_{0}\right)=\bar{N}(r, w+b)+S(r, w) \leqq T(r, w)+S(r, w) ; \\
& \bar{N}\left(r, 0, g_{1}\right)=\bar{N}\left(r, 0, w^{\prime}\right) \leqq T\left(r, w^{\prime}\right) \leqq \frac{m}{n} T(r, w)+S(r, w) ; \\
& m(r, \phi) \leqq \mu T(r, w)+S(r, w) \quad \text { (by Lemma 2(i)) } \\
& S(r)=S(r, w) .
\end{aligned}
$$

Then, from (13), we obtain

$$
m T(r, w) \leqq \mu T(r, w)+T(r, w)+\frac{m}{n} T(r, w)+S(r, w),
$$

that is,

$$
\left(m-\mu-1-\frac{m}{n}\right) T(r, w) \leqq S(r, w),
$$

which is absurd, because $m-\mu-1-\frac{m}{n}$ is positive. This shows that the proposition I holds good.

II. Let $a \neq 0, b$ be meromorphic in $|z|<\infty$. Then the differential equation

$$
\left(w^{\prime}\right)^{n}=a(w+b)^{m} \quad(1 \leqq m \leqq n-1)
$$

does not possess any admissible solutions when $b$ is not constant.

In fact, suppose that (14) admits an admissible solution $w=w(z)$ when $b$ is not constant. Note that, as $b$ is not constant, $w$ is transcendental. We may apply the method used in [9] to prove a part of Theorem A.

Differentiating the equality

we obtain

$$
\left(w^{\prime}(z)\right)^{n}=a(w(z)+b)^{m},
$$

$$
n\left(w^{\prime}\right)^{n-1} w^{\prime \prime}=a^{\prime}(w+b)^{m}+m a(w+b)^{m-1}\left(w^{\prime}+b^{\prime}\right) .
$$


Eliminating $w=w(z)$ from (15) and (16), we have

$$
\left(w^{\prime}\right)^{n-m}\left(n w^{\prime \prime}-\frac{a^{\prime}}{a} w^{\prime}\right)^{m}=m^{m} a\left(w^{\prime}+b^{\prime}\right)^{m}
$$

as $w^{\prime} \not \equiv 0$. We want to show

$$
N\left(r, 0, w^{\prime}\right)=S(r, w) .
$$

When $w^{\prime} \neq 0$ at any point, there is nothing to prove. When $w^{\prime}=0$ at some points, let $z_{0}$ be a zero of $w^{\prime}$ of order $k(\geqq 1)$. Let $s(\geqq 0)$ be the order of zero of $a(z)$ at $z_{0}$ and $t(\geqq 0)$ the order of zero of $b^{\prime}(z)$ at $z_{0}$. (If $z_{0}$ is a pole of $a(z)$ or $b^{\prime}(z)$, we consider that $a(z)$ (or $b^{\prime}(z)$ ) has a zero of order $-s($ or $-t)$ at $z_{0}$.)

(i) The case $a\left(z_{0}\right) \neq 0, \infty$.

In this case, we can easily prove

from (17).

$$
k \leqq t
$$

(ii) The case $a\left(z_{0}\right)=0$ and $b^{\prime}\left(z_{0}\right) \neq \infty$.

$k \leqq t$ or if $k>t$, from (17) we have

and

$$
(n-m) k+m(k-1) \leqq s+m t
$$

$$
k \leqq t+1+s / n .
$$

(iii) The case $a\left(z_{0}\right)=0$ and $b^{\prime}\left(z_{0}\right)=\infty$.

From (17), we have

and so

$$
(n-m) k+m(k-1) \leqq s
$$

(iv) The case $a\left(z_{0}\right)=\infty$.

$$
k \leqq 1+s / n
$$

We can easily see that $k \leqq t$.

From (i)-(iv), we obtain (18):

$$
N\left(r, 0, w^{\prime}\right) \leqq N\left(r, 0, b^{\prime}\right)+\bar{N}(r, 0, a)+N(r, 0, a) / n=S(r, w) .
$$

Next, we wish to prove

In fact, dividing both sides of (17) by $\left(w^{\prime}\right)^{n}$ and then we substitute $v=1 / w^{\prime}$. Noting that

we obtain

$$
m\left(r, 1 / w^{\prime}\right)=S(r, w) .
$$

$$
w^{\prime \prime} / w^{\prime}=-v^{\prime} / v
$$

$$
\left(-n v^{\prime}-\frac{a^{\prime}}{a} v\right)^{m}=m^{m} a\left(1+v b^{\prime}\right)^{m} v^{n},
$$

so that

$$
v^{n+m-1}\left(m^{m} a\left(b^{\prime}\right)^{m} v\right)=Q\left(v, v^{\prime}\right),
$$

$Q\left(v, v^{\prime}\right)$ being differential polynomial in $v$ of degree $n+m-1$. Applying 
LEMMA 3,

That is,

$$
m(r, v)=S(r, v)
$$

$$
m\left(r, 1 / w^{\prime}\right)=S\left(r, w^{\prime}\right),
$$

therefore, by Lemma 4, we obtain (19).

From (18) and (19),

$$
T\left(r, w^{\prime}\right)=S(r, w) .
$$

On the other hand, applying Lemma 2(iii) to (15), we obtain

$$
T(r, w) \leqq \frac{n}{m} T\left(r, w^{\prime}\right)+S\left(r, w^{\prime}\right),
$$

so that from (20),

$$
T(r, w)=S(r, w),
$$

which is absurd. This shows that the proposition II holds good.

III. If the differential equation

$$
\left(w^{\prime}\right)^{n}=a(w+\alpha)^{n} \quad(1 \leqq m \leqq n-1),
$$

where $a(\not \equiv 0)$ is meromorphic in $|z|<\infty$ and $\alpha$ is a constant, possesses an admissible solution, then $n-m$ is a divisor of $n$.

In fact, let $w=w(z)$ be an admissible solution of (22). Substituting $w(z)+\alpha=1 / v(z)$, we obtain

$$
\left(v^{\prime}(z)\right)^{n}=(-1)^{n} a v(z)^{2 n-m} .
$$

This shows that $v(z)$ is an admissible solution of the differential equation

because

$$
\left(v^{\prime}\right)^{n}=(-1)^{n} a v^{2 n-m}
$$

$$
T(r, v)=T(r, w)+O(1) .
$$

Therefore, by a result of Gackstatter and Laine ([3], Satz 6), $n-m$ must be a divisor of $n$.

Combining I, II and III, we obtain Theorem 1.

REMARK 1. When $n-m$ is a divisor of $n$, it is unknown whether (22) possesses an admissible solution or not in the general case. This is closely related with an unsolved problem of Hayman ([6], 1.21). But, specially, if there exist two constants $\sigma>1$ and $M>0$ such that

$$
T(\sigma r, a) \leqq M T(r, a) \quad(r \notin E),
$$

then we can prove that (22) does not admit any admissible solutions, applying a a result of Valiron ([10], p. 33). 
COROLLARY. If the coefficients of (1) are rational, any meromorphic solution in $|z|<\infty$ of (1) is rational when $0 \leqq m \leqq n-1$ ([1], Lemma $1(\mathrm{i})$ ).

Proof. We have only to prove the case when $n-m$ is a divisor of $n$ (including the case $m=0$ ) and the differential equation (1) has the form

$$
\left(w^{\prime}\right)^{n}=a(w+\alpha)^{m}
$$

where $a$ is rational and $\alpha$ is constant. Suppose that this equation admits a transcendental meromorphic solution $w=w(z)$ in $|z|<\infty$, then

$$
u=w^{\prime} /(w+\alpha)^{m / n}=a^{1 / n} .
$$

Both sides of this equality are algebroid functions and

$$
T(r, u)=O(\log r)
$$

as $a$ is rational. On the other hand, $w^{\prime} /(w+\alpha)^{m / n}$ is the derivative of the transcendental algebroid function

$$
\frac{n}{n-m}(w+\alpha)^{(n-m) / n}
$$

so that, applying a result of Valiron ([10]), we obtain

$$
\lim _{r \rightarrow \infty} T(r, u) / \log r=\infty \text {. }
$$

This is a contradiction. Thus, we obtain this corollary.

\section{Order of the solutions}

We note first the following two lemmas.

LEMMA 5. Let $w=w(z)$ be a meromorphic function of order greater than $\rho$ in $|z|<\infty$ and $F$ be the field of meromorphic functions of order at most $\rho$ in $|z|<\infty$, $\rho$ being a nonnegative number. Then $1, w, w^{2}, \cdots, w^{k}(k \geqq 1)$ are linearly independent over $F$.

We can easily prove this by the mathematical induction.

LEMMA 6. Let $A(r)$ and $B(r)$ be positive and increasing functions defined for $r>0$ such that

$$
A(r) \leqq B(r) \quad(r \notin E)
$$

Then, the order of $A(r)$ is not greater than that of $B(r)$.

We can also easily prove this lemma considering the definition of the order of positive increasing function. 
Now, we denote the order of $a$, by $\rho_{j}(j=0,1, \cdots, m)$, where $a$,'s are the coefficients of (1). Suppose that all $\rho$, are finite and put

$$
\rho=\max \left(\rho_{0}, \rho_{1}, \cdots, \rho_{m}\right) \text {. }
$$

Then, we obtain the following

THEOREM 2. When $0 \leqq m \leqq n-1$, any meromorphic solution in $|z|<\infty$ of the differential equation (1) is of order at most $\rho$.

Proof. We shall prove this theorem dividing into two cases.

I. When $2 \leqq m \leqq n-1$, the differential equation (1) does not admit any meromorphic solutions in $|z|<\infty$ of order greater than $\rho$ except when (1) has the form

$$
\left(w^{\prime}\right)^{n}=a_{m}(w+b)^{m},
$$

where $b$ is meromorphic in $|z|<\infty$ of order at most $\rho$.

In fact, suppose that (1) does not have the form (23) and admits a meromorphic solution $w=w(z)$ in $|z|<\infty$ of order greater than $\rho$ for $2 \leqq m \leqq n-1$. We rewrite the righthand side of (1) as in the proof of Theorem $1, \mathrm{I}$ :

$$
\sum_{j=0}^{m} a_{j} w^{\jmath}=a_{m}(w+b)^{m}+\sum_{j=0}^{\mu} b_{j} w^{\jmath} \quad\left(0 \leqq \mu \leqq m-2, b_{\mu} \neq 0\right),
$$

where $b=a_{m-1} / m a_{m}$ and $b$, is a rational function of $a_{\jmath}, a_{m-1}$ and $a_{m}$. By the choice of $b, \mu \leqq m-2$. We note that the orders of $b$ and $b$, are at most $\rho$. Here, we apply Lemma 1 to

$$
g_{0}=-a_{m}(w(z)+b)^{m}, \quad g_{1}=\left(w^{\prime}(z)\right)^{n} \quad \text { and } \quad \phi=\sum_{j=0}^{\mu} b_{j}(w(z))^{\jmath} .
$$

Then,

$$
g_{0}+g_{1}=\psi
$$

By the assumptions and Lemma 5 ,

$$
\phi \not \equiv 0,
$$

and by Lemma 5 and (25), $g_{0}$ and $g_{1}$ are linearly independent over $C$. Now, by Lemma 1 ,

$$
\begin{aligned}
T\left(r, g_{0}\right) \leqq & m(r, \psi)+N\left(r, g_{0}\right)+\bar{N}\left(r, 0, g_{0}\right)+\bar{N}\left(r, 0, g_{1}\right) \\
& +\bar{N}\left(r, g_{0}\right)+\bar{N}\left(r, g_{1}\right)+S(r) .
\end{aligned}
$$

Here, we estimate each term of this inequality.

$$
\begin{gathered}
T\left(r, g_{0}\right) \geqq m T(r, w)-m T(r, b)-T\left(r, a_{m}\right)-O(1) \quad(\text { by Lemma 2(iii)); } \\
m(r, \phi) \leqq \mu m(r, w)+\sum_{j=0}^{\mu} m\left(r, b_{j}\right)+O(1) \quad(\text { by Lemma 2(i) })
\end{gathered}
$$




$$
\leqq \mu T(r, w)+\sum_{j=0}^{\mu} T\left(r, b_{j}\right)+O(1) ;
$$

(29) the order of $N\left(r, g_{0}\right)$ is at most $\rho$.

In fact, as

$$
N\left(r, g_{0}\right) \leqq N\left(r, a_{m}\right)+m N(r, w)+m N(r, b),
$$

we have only to prove that the order of $N(r, w)$ is at most $\rho$. As is remarked in [3], p. 266, $w(z)$ does not have any poles other than those of $a$,'s and it is easily seen that the multiplicities of poles of $w(z)$ are not greater than those of $a$,'s. Hence,

$$
N(r, w) \leqq \sum_{j=0}^{m} N\left(r, a_{j}\right) .
$$

This shows that the order of $N(r, w)$ is at most $\rho$.

$$
\begin{aligned}
\bar{N}\left(r, 0, g_{0}\right) & \leqq \bar{N}(r, 0, w+b)+\bar{N}\left(r, 0, a_{m}\right) \\
& \leqq T(r, w)+T(r, b)+T\left(r, a_{m}\right)+O(1) ;
\end{aligned}
$$

$$
\bar{N}\left(r, 0, g_{1}\right)=\bar{N}\left(r, 0, w^{\prime}\right) \leqq T\left(r, w^{\prime}\right) \leqq \frac{m}{n} T(r, w)+\frac{1}{n} \sum_{j=0}^{m} T\left(r, a_{j}\right)+O(1)
$$

(by (1) and Lemma 2(ii));

(32) the order of $\bar{N}\left(r, g_{0}\right)$ is not greater than $\rho$ (by (29));

(33) the order of $\bar{N}\left(r, g_{1}\right)$ is not greater than $\rho$ because $\bar{N}\left(r, g_{1}\right)=\bar{N}(r, w)$;

$$
S(r)=O(\log T(r, w))+O\left(\sum_{j=0}^{m} \log ^{+} T\left(r, a_{j}\right)\right)+O(\log r) \quad(r \notin E) .
$$

In fact, as

and

we have

$$
T\left(r, g_{0}\right) \leqq m T(r, w)+T\left(r, a_{m}\right)+T(r, b)+O(1)
$$

Further

$$
T(r, b) \leqq T\left(r, a_{m-1}\right)+T\left(r, a_{m}\right)+O(1),
$$

$\log T\left(r, g_{0}\right) \leqq \log T(r, w)+\log ^{+} T\left(r, a_{m-1}\right)+\log ^{+} T\left(r, a_{m}\right)+O(1)$.

$$
\log T\left(r, g_{1}\right) \leqq \log T(r, w)+\sum_{j=0}^{m} \log ^{+} T\left(r, a_{j}\right)+O(1) .
$$

So, from the definition of $S(r)$, we have (34).

Using (26)-(34), we obtain

$$
\begin{aligned}
(m-\mu-1-o(1)) T(r, w) \leqq & N\left(r, g_{0}\right)+\bar{N}\left(r, g_{0}\right)+\bar{N}\left(r, g_{1}\right)+K \sum_{j=0}^{m} T\left(r, a_{j}\right) \\
& +O\left(\sum_{j=0}^{m} \log ^{+} T\left(r, a_{j}\right)\right)+O(\log r) \quad(r \notin E)
\end{aligned}
$$


for $r \rightarrow \infty$, where $K$ is a constant. (Note that $b$,'s are rational in $a_{\jmath}, a_{m-1}$ and $a_{m}$.) As the order of righthand side of this inequality is at most $\rho$ and $m-\mu$ $-1-o(1)>0$, by Lemma 6 , the order of $T(r, w)$ is at most $\rho$. This is a contradiction. This shows that the proposition I holds good.

II. Let $w=w(z)$ be any meromorphic solution in $|z|<\infty$ of

$$
\left(w^{\prime}\right)^{n}=a(w+b)^{m} \quad(0 \leqq m \leqq n-1),
$$

where $a(\not \equiv)$ and $b$ are meromorphic in $|z|<\infty$ of order at most $\rho(<\infty)$. Then the order of $w$ is also at most $\rho$.

As this is trivial when $m=0$, we prove this when $1 \leqq m \leqq n-1$. Suppose that the order of $w$ is greater than $\rho$. As in (17), we have

$$
\begin{gathered}
\left(w^{\prime}\right)^{n-m}\left(n w^{\prime \prime}-\frac{a^{\prime}}{a} w^{\prime}\right)^{m}=m^{m} a\left(w^{\prime}+b^{\prime}\right)^{m} . \\
b^{\prime} \neq 0 .
\end{gathered}
$$

If $b^{\prime} \equiv 0$, that is, if $b$ is a constant, it can be easily seen as in the case of Ozawa ([9], Lemma 2) that the order of $w$ is equal to that of $a$. This is a contradiction to our assumption.

(38) the order of $N\left(r, 0, w^{\prime}\right)$ is at most $\rho$.

The first inequality of $\left(18^{\prime}\right)$ also holds good in this case, and we have (38).

Next, Substituting $w^{\prime}=1 / v$ in (36), we have

$$
v^{n-m}\left(b^{\prime} v+1\right)^{m}=-\frac{1}{m^{m} a}\left(-n \frac{v^{\prime}}{v}-\frac{a^{\prime}}{a}\right)^{m},
$$

so that, as $b^{\prime} \neq \equiv((37))$,

$$
v^{n}=-\frac{1}{\left(b^{\prime}\right)^{m}}\left(\sum_{j=0}^{m-1}\left(\begin{array}{c}
m \\
\jmath
\end{array}\right)\left(b^{\prime}\right)^{\jmath} v^{\jmath+n-m}\right)+m^{-m} a^{-1}\left(b^{\prime}\right)^{-m}\left(-n \frac{v^{\prime}}{v}-\frac{a^{\prime}}{a}\right)^{m} .
$$

From this we obtain by Lemma 2(i)

$$
\begin{aligned}
n m(r, v) \leqq & (n-1) m(r, v)+K T\left(r, b^{\prime}\right)+T(r, a)+O(\log T(r, v)) \\
& +O\left(\log ^{+} T(r, a)\right)+O(\log r) \quad(r \notin E),
\end{aligned}
$$

where $K$ is a constant depending only on $m$. That is,

$$
m(r, v) \leqq K T\left(r, b^{\prime}\right)+T(r, a)+O(\log T(r, v))+O\left(\log ^{+} T(r, a)\right)+O(\log r)
$$

Adding $N\left(r, 0, w^{\prime}\right)$ to both sides of this inequlity, we obtain

$$
(r \notin E) .
$$

$$
(1-o(1)) T\left(r, w^{\prime}\right) \leqq N\left(r, 0, w^{\prime}\right)+K T\left(r, b^{\prime}\right)+T(r, a)+O\left(\log ^{+} T(r, a)\right)+O(\log r)
$$

for $r \rightarrow \infty(r \notin E)$. The order of right-hand side is at most $\rho$ from (38) and the 
assumption, so that by Lemma 6 , the order of $w^{\prime}$ is also at most $\rho$. As the order of $w$ is equal to that of $w^{\prime}$, this is a contradiction to our asumption that the order of $w$ is greater than $\rho$. This shows that the proposition II holds good.

Combining I and II, the proof of Theorem 2 is complete.

EXAMPLES i) The differential equation

$$
\left(w^{\prime}\right)^{n}=e^{z} w-z e^{z}+1
$$

has a solution $w=z$, of which order is smaller than those of coefficients.

ii) The differential equation

$$
\left(w^{\prime}\right)^{n}=w+e^{n z}-e^{z}
$$

has a solution $w=e^{z}$, of which order is equal to the maximum of the orders of coefficients.

iii) The differential equation

$$
\left(w^{\prime}\right)^{n}=\frac{e^{n z}-1}{e^{z}-z} w+\frac{e^{z}-z e^{n z}}{e^{z}-z}
$$

has solutions $w=z$ and $w=e^{z}$. The order of $z$ is smaller than those of coefficients and the order of $e^{z}$ is equal to the maximum of the orders of coefficients.

\section{REFERENCES}

[1] S.B. BANK AND R.P. KaUfman, On the growth of meromorphic solutions of the differential equation $\left(y^{\prime}\right)^{m}=R(z, y)$, Acta Math. 144 (1980), 223-248.

[2] J. Clunie, On integral and meromorphic functions, J. London Math. Soc. 37 (1962), $17-27$.

[3] F. Gackstatter and I. Laine, Zur Theorie der gewöhnlichen Differentialgleichungen im Komplexen, Ann. Polo. Math. 38 (1980), 259-287.

[4] A. A. Gol'Dberg AND I. V. OstrowskiI, The distribution of values of meromorphic functions, Nauka, Moscow, 1970 (in russian).

[5] W.K. Hayman, Meromorphic functions, Oxford Univ. Press, 1964.

[6] W.K. Hayman, Research problems in function theory, Athlone Press, 1967.

[7] A.Z. Mокноn'ко, On the Nevanlinna characteristic of some meromorphic functions, Theory of functions, functional analysis and their applications 14 (1971), 83-87 (in russian).

[8] R. Nevanlinna, Le théorème de Picard-Borel et la théorie des fonctions méromorphes, Gauthier-Villars, Paris, 1929.

[9] M. OzAwA, On a conjecture of Gackstatter and Laine, Kodai Math. J. 6 (1983), 80-87.

[10] G. Valiron, Sur la dérivée des fonctions algébroïdes, Bull. Soc. Math. France 59 (1931), 17-39.

Department of Mathematics

Nagoya Institute of Technology 